\title{
Clinical study in genital herpes: natural Gene- Eden-VIR/Novirin versus acyclovir, valacyclovir, and famciclovir
}

\section{Hanan Polansky \\ Adrian Javaherian \\ Edan Itzkovitz}

The Center for the Biology of Chronic Disease (CBCD), Valley Cottage, NY, USA

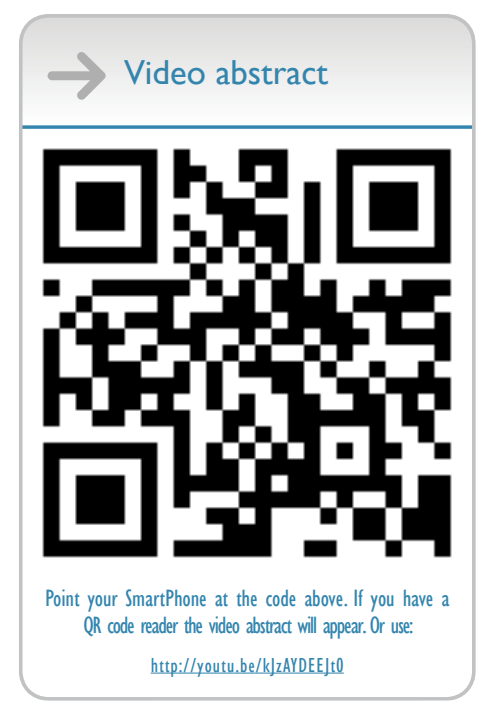

Correspondence: Hanan Polansky The Center for the Biology of Chronic Disease (CBCD), 616 Corporate Way, Suite 2-3665, Valley Cottage, NY 10989, USA

Tel +I 5852509999

Email hpolansky@cbcd.net
This article was published in the following Dove Press journal:

Drug Design, Development and Therapy

29 August 2016

Number of times this article has been viewed

Background: This paper reports the results of a clinical study that tested the effect of suppressive treatment with the botanical product Gene-Eden-VIR/Novirin on the number of genital herpes outbreaks. The results in this study were compared to those published in clinical studies of acyclovir, valacyclovir, and famciclovir.

Methods: The framework was a retrospective chart review. The population included 139 participants. The treatment was one to four capsules of Gene-Eden-VIR/Novirin per day. The duration of treatment was $2-48$ months. The study included three controls recommended by the US Food and Drug Administration (FDA): baseline, no treatment, and dose response.

Results: The treatment decreased the number of outbreaks per year in $90.8 \%$ of the participants. The treatment also decreased the mean number of outbreaks per year from 7.27 and 5.5 in the control groups to 2.39 ( $P<0.0001$ and $P<0.001$, respectively). The treated participants reported no adverse experiences. Out of the 15 tests that compared Gene-Eden-VIR/Novirin to the three drugs, Gene-Eden-VIR/Novirin had superior efficacy in eight tests, inferior efficacy in three tests, and comparable efficacy in four tests. Gene-Eden-VIR/Novirin also had superior safety. Conclusion: The clinical study showed that the natural Gene-Eden-VIR/Novirin decreases the number of genital herpes outbreaks without any side effects. The study also showed that the clinical effects reported in this study are mostly better than those reported in the reviewed studies of acyclovir, valacyclovir, and famciclovir.

Keywords: acyclovir, valacyclovir, famciclovir, natural treatment, genital herpes, outbreaks

\section{Introduction}

Genital herpes is a sexually transmitted viral infection caused by the herpes simplex virus (HSV) type 2 and HSV type 1 (HSV-1). ${ }^{1}$ Genital herpes is often characterized by recurrent outbreaks. The median recurrence rate after the first episode of genital herpes is about four outbreaks per year, with $\sim 40 \%$ of patients having at least six outbreaks and $20 \%$ having more than ten outbreaks per year. ${ }^{2,3}$

Three drugs are currently used in the treatment of recurrent genital herpes: acyclovir (ACV), valacyclovir (VACV, a prodrug of ACV), and famciclovir (FCV, a prodrug of penciclovir). ${ }^{1,4} \mathrm{ACV}$ was approved by the US Food and Drug Administration (FDA) in 1984, VACV in 1995, and FCV in 1994. These drugs use modified nucleosides, or their prodrugs. ${ }^{5}$ The drugs inhibit the activity of the viral DNA polymerase, which is the main replication enzyme of the virus. All drugs are used in both suppressive and episodic therapies. Episodic therapy uses self-administration of the drug during outbreaks, and the main objective is decreasing the duration of the outbreaks. ${ }^{6}$ Clinical studies have shown that episodic treatment with these drugs shortens the time to lesion 
healing by $1-2$ days. $^{7-9}$ Suppressive therapy involves daily administration of the drug before the onset of an outbreak. The most common objective of suppressive therapy is decreasing the number of outbreaks. ${ }^{10}$ Clinical studies have demonstrated that suppressive treatment with these drugs for a period of 4-12 months decreases the number of outbreaks such that about half of patients remain recurrence-free and the other half show a $70 \%-80 \%$ decrease in the frequency of their outbreaks. ${ }^{11,12}$

Gene-Eden-VIR/Novirin is a patented botanical product that consists of five natural ingredients: quercetin $100 \mathrm{mg}$, green tea extract $150 \mathrm{mg}$, cinnamon extract $50 \mathrm{mg}$, selenium $100 \mu \mathrm{g}$, and licorice extract $25 \mathrm{mg}$. Gene-Eden-VIR/Novirin was developed to target latent viruses, and diminish their deleterious effect on the host, as explained by the Microcompetition theory. ${ }^{13-15}$ Gene-Eden-VIR/Novirin was introduced in the marketplace at the end of 2009. A post-marketing clinical study conducted at the Center for the Biology of Chronic Disease showed that Gene-Eden-VIR/Novirin is antiviral. ${ }^{16}$ Another post-marketing clinical study showed that Gene-Eden-VIR/Novirin safely decreased the feeling of fatigue in individuals infected with a latent virus. ${ }^{17}$

This paper reports the results of a post-marketing clinical study that tested the effect of suppressive treatment with Gene-Eden-VIR/Novirin on the frequency of genital herpes outbreaks, or the number of outbreaks per period. The paper also compares the clinical effects of Gene-Eden-VIR/Novirin to those of ACV, VACV, and FCV.

\section{Materials and methods Objective}

The objective of this clinical study (ClinicalTrials.gov Identifier: NCT02715752) was to test the effect of Gene-Eden-VIR/ Novirin on the frequency of genital herpes outbreaks.

\section{Framework}

The framework was a retrospective chart review. The company that sells Gene-Eden-VIR/Novirin provides a service to the customers who purchase the products. The service consists of tracking the changes in the customers' health while using the products. The company is using a questionnaire called the Natural Origin Treatment Clinical Questionnaire (NotCiq). The data are collected over the phone by professional interviewers in a single session. The NotCiq is a patient-reported outcome (PRO) instrument. The charts produced in these sessions were analyzed in this study. More details on the NotCiq questionnaire are available in our previous study. ${ }^{16}$

\section{Randomization}

Randomly selected charts, collected during a 2-month period, October and November 2015, were analyzed. The two months were randomly selected.

\section{Treatment}

Gene-Eden-VIR/Novirin is a patented botanical product that consists of five natural ingredients: quercetin $100 \mathrm{mg}$, green tea extract $150 \mathrm{mg}$, cinnamon extract $50 \mathrm{mg}$, selenium $100 \mu \mathrm{g}$, and licorice extract $25 \mathrm{mg}$. The treatment was one to four capsules of Gene-Eden-VIR/Novirin per day.

The duration of treatment ranged from 2 to 48 months. The mean duration of treatment was 12 months.

The treatment group included 119 participants. The study included three FDA-recommended controls: baseline control, consisting of 119 participants; no-treatment control, consisting of 20 participants; and dose-response concurrent control, consisting of 119 participants. The baseline control included participants "before" treatment or pretreatment. The no-treatment control included participants who had just purchased Gene-Eden-VIR/Novirin, and had not yet started taking the treatment.

\section{Outcome measures}

The NotCiq for genital herpes has several sections: a section on gender, age, and ethnicity of the participants; a section on the duration of treatment, dosage, and adverse experiences; a section on diagnosis and type of symptoms; a section on the frequency of symptoms; and a section on blood pressure and body weight. The questionnaire uses both open- and closed-ended questions. The answers to the closed-ended questions are on a scale of 1-7. The answers to the NotCiq were collected in phone interviews by four independent interviewers, who were specialized in outbound call services. The interviewers were blinded to the objective of the study.

\section{Efficacy}

The primary end point was the number of outbreaks per period. Secondary end points included the time to first recurrence, the percentage of recurrence-free patients, the percentage of prevented or delayed recurrences, and the number of participants who had over a $50 \%$ reduction in their recurrence rate.

\section{Safety}

The participants' reports of adverse events were collected and analyzed. 


\section{Population}

Participants who were using Gene-Eden-VIR/Novirin for other purposes, such as treatment for cancer, chronic diseases, and hypertension, were excluded.

Participants who were concurrently taking antiviral medications, including ACV, VACV, and FCV, as suppressive treatment were also excluded. The final list of participants consisted of 139 men and women aged $\geq 18$ years with at least one genital herpes outbreak per year.

\section{Ethical consideration}

The study was approved by the Salus Institutional Review Board. The approval included a complete waive to obtain patient's informed consent, due to the nature of the study.

\section{Statistical analysis}

The analysis was based on the intent-to-treat (all participants randomized) population. We analyzed the time to first recurrence of a genital herpes outbreak using the Kaplan-Meier product limit method, and the differences between the curves were evaluated by log-rank, Wilcoxon, and Tarone-Ware tests. We also calculated the delta, that is, the difference in scores between pretreatment and during treatment. Then, we tested the statistical difference between the deltas. Statistical analysis was performed using a two-tail $t$-test assuming unequal variances. $P \leq 0.05$ was considered as statistically significant.

\section{Results}

\section{Patient demographics and baseline characteristics}

A total of 139 participants were included in the study. Table 1 summarizes the demographics and clinical characteristics of these participants (Table 1).

\section{Efficacy}

Primary efficacy end points

Out of the 119 participants in the treatment group, 108 $(90.8 \%)$ reported a decrease in the number of recurrences per year. The mean number of recurrences per year decreased from 7.27 and 5.5 in the pretreatment and no-treatment controls, respectively, to 2.39 in the treatment group (67\% and $57 \%, P<0.0001$ and $P<0.001$, respectively) (Table 2 ).

We also tested the effect of different dosages (Table 3). Because participants with a greater number of outbreaks had a tendency to take a higher dosage of Gene-Eden-VIR/ Novirin, we selected participants with a similar number of recurrences per year. Specifically, we selected those with two to four recurrences. The data included 42 such participants,
Table I Demographics and clinical characteristics of the participants

\begin{tabular}{|c|c|}
\hline Age average (years) & 51 \\
\hline \multicolumn{2}{|l|}{ Age (years), number (\%) } \\
\hline $20-40$ & $26(21.8 \%)$ \\
\hline $4 I-50$ & $28(23.5 \%)$ \\
\hline $5 I-60$ & $37(31.1 \%)$ \\
\hline $61-80$ & $28(23.5 \%)$ \\
\hline \multicolumn{2}{|l|}{ Gender, number (\%) } \\
\hline Male & $64(53.8 \%)$ \\
\hline Female & $55(46.2 \%)$ \\
\hline \multicolumn{2}{|l|}{ Race, number (\%) } \\
\hline African American & $30(25.2 \%)$ \\
\hline Caucasian & $67(56.3 \%)$ \\
\hline Hispanic & $12(10.1 \%)$ \\
\hline Other & $10(8.4 \%)$ \\
\hline Years since diagnosis by physician & $\begin{array}{l}0.5-40 \text { (range), } 10.5 \\
\text { (mean), } 5 \text { (median) }\end{array}$ \\
\hline Years since initial episode & $\begin{array}{l}0.5-48 \text { (range), } 13.8 \\
\text { (mean), } 9.5 \text { (median) }\end{array}$ \\
\hline Percentage diagnosed by physician & $80.7 \%$ \\
\hline $\begin{array}{l}\text { Percentage who received a blood test } \\
\text { (out of those diagnosed by a physician) }\end{array}$ & $65.6 \%$ \\
\hline \multicolumn{2}{|l|}{ Symptoms of infection, number (\%) } \\
\hline Genital blisters/ulcers & $100(84.0 \%)$ \\
\hline Anal blisters/ulcers & $22(18.5 \%)$ \\
\hline Burning feeling while urinating & $28(23.5 \%)$ \\
\hline Local pain & $84(70.6 \%)$ \\
\hline Genital discharge & $12(10.1 \%)$ \\
\hline General discomfort & $62(52.1 \%)$ \\
\hline Light sensitivity & $23(19.3 \%)$ \\
\hline Genital tingling sensation & 78 (65.5\%) \\
\hline Genital itching sensation & $81(68.1 \%)$ \\
\hline Flu-like symptoms & 55 (46.2\%) \\
\hline Irritation (including burning) & $21(17.6 \%)$ \\
\hline Redness & $18(15.1 \%)$ \\
\hline Fatigue & $4(3.4 \%)$ \\
\hline Lower extremity pain & $10(8.4 \%)$ \\
\hline \multicolumn{2}{|l|}{ Duration of treatment (months), number (\%) } \\
\hline $2-3$ & $2 \mathrm{I}(17.8 \%)$ \\
\hline $4-6$ & $25(21.2 \%)$ \\
\hline $9-12$ & $29(24.6 \%)$ \\
\hline $16-24$ & $29(24.6 \%)$ \\
\hline $26-48$ & 14 (II.9\%) \\
\hline
\end{tabular}

18 who took one capsule and 24 who took two capsules per day. The mean number of outbreaks per year before treatment was similar in both dosage groups (3.11 and 3.08, respectively; $P=0.45$ ). We then analyzed the number of outbreaks per year following treatment in both groups. The mean number of recurrences per year decreased to 0.9 and 0.5 in the group that took one and two capsules, respectively $(P=0.08)$. These results had borderline significance for the existence of a dose effect. We also tested for the existence of a dose effect from another direction. We analyzed the deltas for the two dosage groups by performing a Student's $t$-test on all 
Table 2 Summary of efficacy end points in the current study

\begin{tabular}{|c|c|c|c|c|c|c|}
\hline Treatment & $\mathbf{N}$ & $\begin{array}{l}\text { Mean number of recurrences } \\
\text { per year }\end{array}$ & $\begin{array}{l}\text { Time to first } \\
\text { recurrence }^{a} \text { (days) }\end{array}$ & $\%$ recurrence-free & $\begin{array}{l}\% \text { with decrease } \\
\text { in recurrence }\end{array}$ & $\begin{array}{l}\% \text { with }>\mathbf{5 0} \% \\
\text { reduction }\end{array}$ \\
\hline $\begin{array}{l}\text { No-treatment } \\
\text { control }\end{array}$ & 20 & 5.5 & 105.01 & $0.0 \%$ & - & - \\
\hline $\begin{array}{l}\text { Pretreatment } \\
\text { control }\end{array}$ & 119 & $7.27\left(3.2^{\mathrm{b}}\right)$ & 103.91 & $0.0 \%$ & $90.8 \%$ & $73.1 \%$ \\
\hline Treatment & 119 & $\begin{array}{l}2.39\left(5.7^{b}\right)(P<0.000 \mathrm{I}, \text { pretreatment }) \\
(P<0.00 \mathrm{I}, \text { no treatment })\end{array}$ & $\begin{array}{l}537.34 \text { ( } P<0.0001 \text {, } \\
\text { both controls) }\end{array}$ & $\begin{array}{l}42.0 \%(P<0.000 \mathrm{I} \\
\text { both controls })\end{array}$ & & \\
\hline
\end{tabular}

Notes: The participants had at least one genital herpes recurrence per year. ${ }^{a}$ Using Kaplan-Meier. ${ }^{b}$ Results from the two questions testing internal consistency $(P<0.000 \mathrm{I})$.

119 participants. The mean decrease in number of recurrences per year (the delta) was 4.2 and 5.9 for one and two capsules, respectively $(P=0.088)$. This test resulted in a similar statistical significance for the existence of a dose effect.

We then tested the effect of different durations of treatment (Table 4). A common inclusion criterion of the clinical studies that tested drugs against genital herpes is at least six genital herpes outbreaks per year. To test for a duration-oftreatment effect, we also used this criterion. The data included 54 such participants. After examining these participants, we removed three outliers from the analysis. These participants had constant outbreaks with no remission. We observed that these participants responded excessively fast to the treatment, and therefore, distorted the duration-of-treatment analysis. The results showed a statistically significant difference in the delta values between those participants who took Gene-Eden-VIR/Novirin for 2-6 months and those who took the treatment for $>6$ months ( 3.8 vs 8.5 , respectively; $P<0.01)$. These results showed the existence of a durationof-treatment effect.

We also analyzed the effect of age on the decrease in the number of recurrences per year. The results showed a negative trend. That is, older people had a smaller decrease in the number of recurrences per year. However, this trend was not statistically significant.

To test the internal consistency of the participants, the following two questions were included: "How often did the symptoms appear before (after) taking Gene-EdenVIR/Novirin?", which were scored on a scale from 1 to 7 , where 1 was "very often" and 7 was "not at all". The mean scores were 3.2 and 5.7 for the questions regarding "before" and "after" taking Gene-Eden-VIR/Novirin, respectively $(P<0.0001)$ (Table 2). The results showed that both the measures of frequency, the number of recurrences per year and the scale between "very often" and "not at all", generated consistent results.

We also tested for the effect of diagnosis on the results (Table 5). The data included two types of diagnoses: diagnosis by a physician and self-diagnosis. The mean number of recurrences per year was 7.32 and 2.40 during the pretreatment and treatment period, respectively $(P<0.0001)$, in participants diagnosed by a physician, and 7.04 and 2.39 , respectively $(P<0.001)$, in self-diagnosed participants. There was no statistically significant difference between the two groups for the two periods ( $P=0.4$ in the pretreatment group and $P=0.5$ in the treatment group).

\section{Secondary efficacy end points}

The time to first recurrence was calculated using the KaplanMeier product limit method. The mean survival time, that is, the number of days to first recurrence, was 103.91 and 105.01 during the pretreatment and no-treatment periods, respectively, and 537.34 days during the treatment period $(P<0.0001$ for treatment vs each control). These results

Table 3 Summary of dosage effect in participants with two to four genital herpes outbreaks per year pretreatment

\begin{tabular}{lllll}
\hline Dosage & N & $\begin{array}{l}\text { Mean number of outbreaks } \\
\text { per year (pretreatment) }\end{array}$ & $\begin{array}{l}\text { Mean number of outbreaks } \\
\text { per year (posttreatment) }\end{array}$ & $\begin{array}{l}\text { Mean decrease in number } \\
\text { of outbreaks per year }(\Delta)\end{array}$ \\
\hline GEV/NV I/day & 18 & 3.11 & 0.9 & - \\
GEV/NV 2/day & 24 & 3.08 & 0.5 & - \\
& & $P=0.45$ & $P=0.08$ & - \\
GEV/NV I/day & 36 & - & - & 4.2 \\
GEV/NV 2/day & 61 & - & - & 5.9 \\
& & & & $P=0.088$ \\
\hline
\end{tabular}

Abbreviation: GEV/NV, Gene-Eden-VIR/Novirin. 
Table 4 Summary of duration-of-treatment effect in participants with at least six genital herpes outbreaks per year pretreatment

\begin{tabular}{lll}
\hline $\begin{array}{l}\text { Duration of } \\
\text { treatment (months) }\end{array}$ & $\mathbf{N}$ & $\begin{array}{l}\text { Mean decrease in number } \\
\text { of outbreaks per year }(\Delta)\end{array}$ \\
\hline $2-6$ & 19 & 3.8 \\
$>6$ & 32 & 8.5 \\
& $P<0.01$ \\
\hline
\end{tabular}

showed that Gene-Eden-VIR/Novirin prolonged the time to first recurrence. Figure 1 clearly shows that treatment with Gene-Eden-VIR/Novirin increased the time to first recurrence in those with at least one recurrence per year (Figure 1A), at least six recurrences per year (Figure 1B), and at least eight recurrences per year (Figure 1C). Figure 1 also shows that treatment with Gene-Eden-VIR/Novirin decreased the frequency of genital herpes outbreaks (Figure 1D).

In both the pretreatment and no-treatment controls, zero participants were recurrence-free (Table 2). After 1 year of treatment, $42 \%$ were recurrence-free $(P<0.0001)$. Also, Gene-Eden-VIR/Novirin prevented or delayed $90.8 \%$ of the recurrences, and 87 of 119 (73.1\%) participants had over a $50 \%$ reduction in the recurrence rate.

\section{Safety}

There were no reports of adverse events from taking GeneEden-VIR/Novirin. In addition, there were no statistically significant changes in body weight. Also, out of the 119 participants, 23 reported suffering from high blood pressure before treatment. Out of these 23 participants, 15 reported no change in their blood pressure, and eight, an improvement, that is, a decrease in their blood pressure. A statistical analysis showed that Gene-Eden-VIR/Novirin significantly lowered the participants' blood pressure $(P<0.01)$.

\section{Gene-Eden-VIR/Novirin vs leading drugs}

The following section compares the clinical effects of Gene-Eden-VIR/Novirin with those reported in published clinical studies that tested the three leading drugs approved by the FDA for the treatment of genital herpes, specifically $\mathrm{ACV}, \mathrm{VACV}$, and FCV.

Table 5 Summary of the effect of self-diagnosis vs diagnosis by a physician on the mean number of recurrences per year

\begin{tabular}{|c|c|c|c|}
\hline $\begin{array}{l}\text { Diagnosis } \\
\text { method }\end{array}$ & $\mathbf{N}$ & $\begin{array}{l}\text { Mean number of } \\
\text { outbreaks per year } \\
\text { (pretreatment) }\end{array}$ & $\begin{array}{l}\text { Mean number of } \\
\text { outbreaks per year } \\
\text { (posttreatment) }\end{array}$ \\
\hline Self-diagnosis & 23 & 7.04 & $2.39(P<0.001)$ \\
\hline $\begin{array}{l}\text { Diagnosis by } \\
\text { physician }\end{array}$ & 96 & 7.32 & $2.40(P<0.0001)$ \\
\hline
\end{tabular}

In order to find the published clinical studies that tested $\mathrm{ACV}, \mathrm{VACV}$, and FCV as a chronic suppressive treatment for genital herpes, we conducted a systematic search of the literature for original research articles indexed in PubMed (MEDLINE) database. We searched for randomized controlled trials that tested ACV, VACV, or FCV as a treatment for genital herpes. In the computer searches, we used the following title/abstract words: "genital", "herpes", "acyclovir", "valacyclovir", "valaciclovir", and "famciclovir". We did not use date restrictions. We included all articles that evaluated the frequency of genital herpes outbreaks before and after oral antiviral therapy in immunocompetent individuals, where the population included both adult men and nonpregnant women. We found five clinical studies. ${ }^{10,12,18-20}$

\section{Efficacy}

The comparative analysis of Gene-Eden-VIR/Novirin efficacy utilized three sets of studies. The first set consisted of three studies: Douglas et al, ${ }^{18}$ Reitano et al, ${ }^{12}$ and Mertz et al ${ }^{19}$ (Table 6). The participants in these studies had at least six recurrences of genital herpes per year. The second set consisted of one study: Patel et al ${ }^{20}$ (Table 7). The participants in this study had at least eight recurrences of genital herpes per year. The third set also consisted of one study: Corey et $\mathrm{a}^{10}$ (Table 8 ). The participants in this study had less than ten recurrences of genital herpes per year.

To summarize, out of 15 tests, Gene-Eden-VIR/Novirin had superior efficacy in eight tests, inferior efficacy in three tests, and comparable efficacy in four tests (Table 9). This indicates that Gene-Eden-VIR/Novirin has comparable-tosuperior efficacy compared to ACV, VACV, and FCV in decreasing the frequency of genital herpes outbreaks.

\section{Safety}

Douglas et $\mathrm{al}^{18}$ observed adverse events in some of their patients, including diarrhea, headache, weight loss, nausea or vomiting, abdominal pain, insomnia, fever, vertigo, and anorexia. Reitano et al ${ }^{12}$ observed mild adverse events, the most frequent of which were headache, rhinitis, infection, and flu-like syndrome. Sixty serious adverse experiences were reported by 48 patients during the Reitano et al study, the most common being depression. Mertz et al ${ }^{19}$ observed that the most common adverse experiences in FCV-treated subjects were headache, nausea, and diarrhea. Patel et $\mathrm{al}^{20}$ observed that most adverse experiences were of mild intensity, and the most common were headache and nausea. Finally, Corey et $\mathrm{al}^{10}$ observed that the frequency of adverse effects was similar to those reported in the study by Patel et al. 

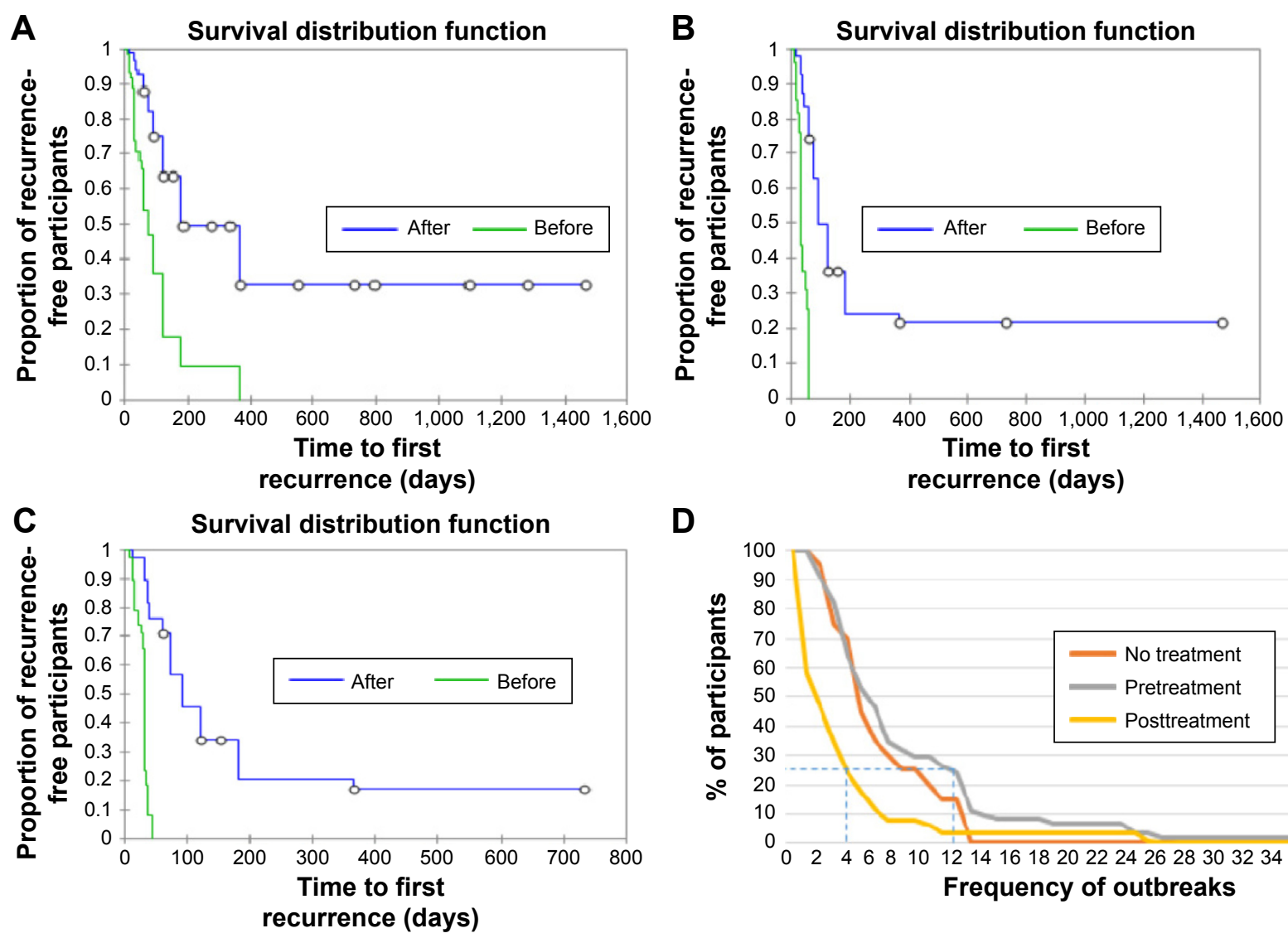

D

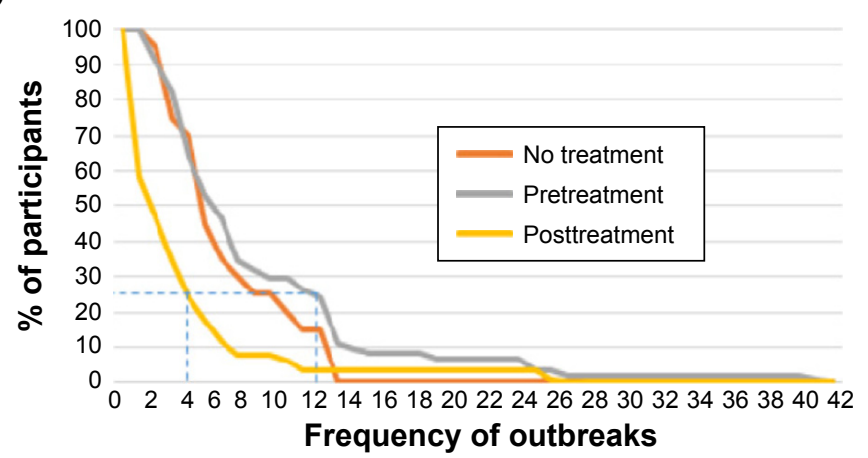

Figure I Graphical presentations of the Gene-Eden-VIR/Novirin effect.

Notes: Kaplan-Meier plots of time to first genital herpes recurrence in the current study from participants with a history of $(\mathbf{A})$ at least one recurrence per year, (B) at least six recurrences per year, and (C) at least eight recurrences per year. (D) Percentage of participants versus frequency of recurrences.

The most frequent adverse effects reported in the product insert for suppressive therapy were the following: nausea, diarrhea, and headache for ACV; headache, nausea, and abdominal pain for VACV; and headache, nausea, and dizziness for FCV.

In addition, the literature reports non-common adverse events for these three drugs. For instance, Yavuz et al reported that a 78-year-old female with normal baseline renal function, and no contributing possible nephrotoxic factors, developed irreversible renal dysfunction after oral ACV treatment. ${ }^{21}$ Becker et al reported that a patient developed rapidly progressive acute renal failure with concomitant mental status changes following treatment with high-dose parenteral ACV. ${ }^{22}$ Another uncommon but serious side effect of ACV treatment is neurotoxicity that may lead to confusion, hallucinations, seizures, and obtundation. ${ }^{23}$

Le Cleach et al conducted a meta-analysis of 26 clinical studies that tested the effectiveness and safety of the three oral antiviral drugs, ACV, FCV, and VACV. ${ }^{24}$ They found that withdrawals due to harms were reported in only eight or
$31 \%$ of the studies. In these studies, 31 withdrawals due to harms were reported in the antiviral groups, in comparison to 14 in the placebo or no-treatment groups. In addition, only four or $16 \%$ of the studies reported safety data in the form of the total number of adverse events. Overall, these four studies reported 331 adverse events in 561 participants (59\%) in the antiviral groups, including three serious adverse events (one hypertension crisis, one intestinal obstruction, and one angor), compared to 115 adverse events in 291 participants in the placebo or no-treatment groups (40\%), including three serious adverse events (two renal signs and one fatal pneumonia).

Lam et al analyzed a cohort of 76,269 patients who received ACV or VACV, and 84,646 who received FCV. ${ }^{25}$ The results showed that $0.27 \%$ of the patients were hospitalized with acute kidney injury following treatment with ACV or VACV, and $0.28 \%$ following treatment with FCV.

In our study, there were no reports of adverse events following treatment with Gene-Eden-VIR/Novirin. Furthermore, there were no statistically significant changes in body weight. 
Table 6 Summary of efficacy end points in the current study vs the studies by Douglas et al, Reitano et al, and Mertz et al

\begin{tabular}{|c|c|c|c|c|c|c|c|}
\hline Study & Treatment & $\mathbf{N}$ & $\begin{array}{l}\text { Time to first } \\
\text { recurrence }^{a} \\
\text { (days) }\end{array}$ & $\begin{array}{l}\% \text { with } \\
>\mathbf{5 0} \% \\
\text { reduction }^{\mathrm{b}} \\
\end{array}$ & $\begin{array}{l}\text { Mean } \\
\text { monthly } \\
\text { recurrence }\end{array}$ & $\begin{array}{l}\text { \% recurrence- } \\
\text { free }^{\mathrm{b}}\end{array}$ & $\begin{array}{l}\text { Mean \% } \\
\text { reduction in } \\
\text { recurrence }\end{array}$ \\
\hline \multirow[t]{4}{*}{ Douglas et $\mathrm{al}^{18}$} & Placebo & 47 & 18 & $58.30 \%$ & 0.86 & $0.0 \%$ & - \\
\hline & ACV $200 \mathrm{mg} 2 \times$ & 51 & $>120(P<0.001)$ & & $0.14(P<0.001)$ & $27.4 \%(P<0.00 I)$ & - \\
\hline & ACV $200 \mathrm{mg} 5 \times$ & 45 & $>120(P<0.001)$ & & $0.13(P<0.001)$ & $35.6 \%(P<0.00 I)$ & - \\
\hline & Average (treatment) & & $120(\Delta=102)$ & & $0.135(\Delta=0.725)$ & $31.5 \%(\Delta=31.5 \%)$ & \\
\hline \multirow[t]{7}{*}{ Reitano et al $^{12}$} & Placebo & 134 & - & - & - & $5.0 \%$ & - \\
\hline & VACV $250 \mathrm{mg} 2 \times$ & 274 & - & & - & $34.0 \%(P<0.001)$ & $78 \%-79 \%$ \\
\hline & VACV I g IX & 269 & - & & - & $34.0 \%(P<0.001)$ & $78 \%-79 \%$ \\
\hline & VACV $500 \mathrm{mg} \mathrm{IX}$ & 266 & - & & - & $28.0 \%(P<0.00 I)$ & $71 \%$ \\
\hline & VACV $250 \mathrm{mg}$ IX & 269 & - & & - & $15.0 \%(P=0.03)$ & $54 \%$ \\
\hline & ACV $400 \mathrm{mg} 2 \times$ & 267 & - & & - & $35.0 \%(P<0.00 I)$ & $78 \%-79 \%$ \\
\hline & Average (treatment) & & & & & $\begin{array}{l}27.8 \%(\Delta=22.8 \% \\
\text { VACV; } \Delta=30.0 \% \\
\text { ACV) }\end{array}$ & $72.1 \%$ \\
\hline \multirow[t]{7}{*}{ Mertz et a $\left.\right|^{19}$} & Placebo & 64 & 82 & - & 0.27 & $6.3 \%$ & - \\
\hline & FCV $125 \mathrm{mg} \mathrm{IX}$ & 60 & $114(P=0.22)$ & & 0.22 & $\begin{array}{l}\text { No significant } \\
\text { increase }\end{array}$ & - \\
\hline & FCV $125 \mathrm{mg} 2 \times$ & 65 & $>120(P=0.03)$ & & 0.16 & $23.1 \%(P<0.04)$ & - \\
\hline & FCV $250 \mathrm{mg} \mathrm{IX}$ & 61 & $>120(P=0.07)$ & & 0.23 & $21.3 \%(P<0.05)$ & - \\
\hline & FCV $250 \mathrm{mg} 2 \times$ & 64 & $>120(P<0.001)$ & & $0.08(P=0.002)$ & $47.0 \%(P<0.001)$ & - \\
\hline & FCV $500 \mathrm{mg} \mathrm{IX}$ & 61 & $>120(P=0.06)$ & & 0.15 & $\begin{array}{l}\text { No significant } \\
\text { increase }\end{array}$ & - \\
\hline & Average (treatment) & & I I $8.8(\Delta=36.8)$ & & $0.168(\Delta=0.102)$ & $30.5 \%(\Delta=24.2 \%)$ & \\
\hline \multirow[t]{2}{*}{ Current study } & GEV/NV pretreatment & 55 & 38 & $69.10 \%$ & 1.02 & $0.0 \%$ & $66 \%$ \\
\hline & GEV/NV treatment & 55 & $\begin{array}{l}238(P<0.000 \mathrm{I}) \\
(\Delta=200)\end{array}$ & & $\begin{array}{l}0.35(P<0.000 \mathrm{I}) \\
(\Delta=0.67)\end{array}$ & $\begin{array}{l}27.3 \%(P<0.000 \mathrm{I}) \\
(\Delta=27.3 \%)\end{array}$ & \\
\hline
\end{tabular}

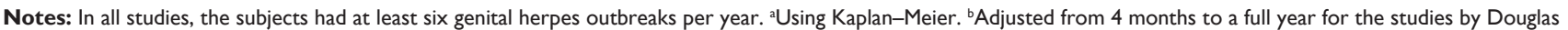
et al and Mertz et al. The $P$-values are taken from the original study.

Abbreviations: ACV, acyclovir; VACV, valacylovir; FCV, famciclovir; GEV/NV, Gene-Eden-VIR/Novirin.

\section{Discussion}

Our study showed that suppressive treatment with Gene-EdenVIR/Novirin decreased the frequency of genital herpes outbreaks. The results also showed the existence of a dose effect and a duration-of-treatment effect. Specifically, the results showed that treatment with two capsules per day decreased the frequency of genital herpes outbreaks more than treatment with one capsule per day, and that treatment for $>6$ months decreased the frequency of outbreaks more than treatment for 2-6 months. Finally, the results showed that suppressive treatment with Gene-Eden-VIR/Novirin has no side effects; that is, suppressive treatment with Gene-Eden-VIR/Novirin is safe.

The study showed that the clinical effects of Gene-EdenVIR/Novirin observed in this clinical study are mostly better than those reported in the reviewed clinical studies that tested ACV, VACV, and FCV (Table 9).

Table 7 Summary of efficacy end points in the current study vs the study by Patel et al

\begin{tabular}{|c|c|c|c|c|c|}
\hline Study & Treatment & $\mathbf{N}$ & $\begin{array}{l}\text { Time to first } \\
\text { recurrence }^{\mathrm{a}} \text { (days) }\end{array}$ & $\begin{array}{l}\% \text { recurrence- } \\
\text { free }^{b}\end{array}$ & $\begin{array}{l}\% \text { with decrease } \\
\text { in recurrence }{ }^{c}\end{array}$ \\
\hline \multirow[t]{2}{*}{ Patel et $\mathrm{al}^{20}$} & Placebo & $94 / 66^{d}$ & 20 & $0.0 \%$ & $85 \%$ \\
\hline & VACV $500 \mathrm{mg} \mathrm{IX}$ & $288 / 198^{a}$ & $\begin{array}{l}>I I 2(P<0.000 I) \\
(\Delta=92)\end{array}$ & $33.0 \%(\Delta=33.0 \%)$ & \\
\hline \multirow[t]{2}{*}{ Current study } & GEV/NV pretreatment & 38 & 28 & $0.0 \%$ & $89 \%$ \\
\hline & GEV/NV treatment & 38 & $\begin{array}{l}\mid 45(P<0.000 \mathrm{I}) \\
(\Delta=|| 7)\end{array}$ & $\begin{array}{l}24.0 \%(P<0.00 \mathrm{I}) \\
(\Delta=24.0 \%)\end{array}$ & \\
\hline
\end{tabular}

Notes: In all studies, the subjects had at least eight genital herpes outbreaks per year. a Using Kaplan-Meier. ${ }^{\mathrm{b}} \mathrm{Adjusted}$ from 4 months to a full year for the Patel et al study. 'Note that $85 \%$ is for a 4 -month period and $89 \%$ is for a 12 -month period. 'Patel et al used 94 (placebo) and 288 (treatment) for calculating the percentage of recurrencefree subjects and percentage of subjects with a decrease in recurrence, respectively. In contrast, Patel et al used 66 (placebo) and 198 (treatment) subjects for calculating the median time to first recurrence.

Abbreviations: GEV/NV, Gene-Eden-VIR/Novirin; VACV, valacyclovir. 
Table 8 Summary of efficacy end point in the current study vs the study by Corey et al

\begin{tabular}{lllll}
\hline Study & Treatment & N & \% recurrence-free & Mean monthly recurrence $^{\text {b }}$ \\
\hline Corey et al & Placebo & $74 I$ & $23 \%$ & 0.4 \\
& VACV $500 \mathrm{mg} \mathrm{I} \times$ & 743 & $53 \%(P<0.00 \mathrm{I})(\Delta=30 \%)$ & $0.1 \mathrm{I}(P<0.00 \mathrm{I})(\Delta=0.29)$ \\
Current study & GEV/NV pretreatment & 84 & $0 \%$ & 0.32 \\
& GEV/NV treatment & 84 & $49 \%(P<0.000 \mathrm{I})(\Delta=49 \%)$ & $0.1 \mathrm{I}(P<0.000 \mathrm{I})(\Delta=0.2 \mathrm{I})$ \\
\hline
\end{tabular}

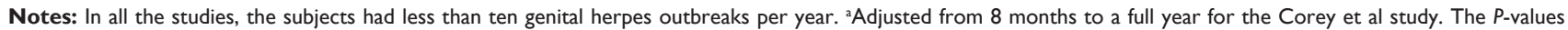
are taken from the original study. bince the distribution of the number of recurrences per patient is not mentioned in Corey et al, it seems that their sample includes many participants with eight or nine recurrences per year, while our participants include many more participants in the 3-5 range. As a result, their mean monthly recurrence in the placebo group is higher than our mean monthly recurrence in the pretreatment group. This initial condition prevents Gene-Eden-VIR/Novirin from showing its full efficacy in this specific sample.

Abbreviations: GEV/NV, Gene-Eden-VIR/Novirin; VACV, Valacyclovir.

The argument for large sample sizes, randomization, independent verification by different laboratories, etc, is to even out specific peculiarities, or confounding factors, inherent to any specific setting. The same result under dissimilar settings is considered reliable. We compared Gene-Eden-VIR/Novirin to $\mathrm{ACV}, \mathrm{VACV}$, and FCV using results reported in five studies. These studies treated diverse populations under different conditions. The comparison showed that Gene-Eden-VIR/Novirin has comparable-to-superior efficacy. Since the conditions in these studies were different, we believe that the conclusion is, most likely, robust. In other words, the observed comparableto-superior efficacy is not an artifact of any specific condition; that is, it has strong external validity.

Table 9 Efficacy of Gene-Eden-VIR/Novirin vs ACV, VACV, and FCV

\begin{tabular}{lll}
\hline Efficacy end point & $\begin{array}{l}\text { Superiority } \\
\text { of efficacy }\end{array}$ & Source \\
\hline Mean number of & GEV/NV $>$ VACV & Mertz et al ${ }^{19}$ \\
recurrences per month & GEV/NV $<$ VACV & Corey et al ${ }^{10}$ \\
& GEV/NV $=$ ACV & Douglas et al ${ }^{18}$ \\
Percentage recurrence-free & GEV/NV $>$ VACV & Corey et al ${ }^{8}$ \\
& GEV/NV $>$ VACV & Reitano et al ${ }^{12}$ \\
& GEV/NV $=$ ACV & Reitano et al ${ }^{12}$ \\
& GEV/NV $<$ ACV & Douglas et al $\left.\right|^{18}$ \\
& GEV/NV $>$ FCV & Mertz et al ${ }^{19}$ \\
& GEV/NV $<$ VACV & Patel et al ${ }^{10}$ \\
Time to first recurrence & GEV/NV $>$ FCV & Mertz et al ${ }^{19}$ \\
& GEV/NV $>$ VACV & Patel et a ${ }^{20}$ \\
& GEV/NV $>$ ACV & Douglas et al $\left.\right|^{18}$ \\
Mean percent reduction in & GEV/NV $=$ VACV & Reitano et al ${ }^{12}$ \\
recurrence & & \\
Percentage with a $>50 \%$ & GEV/NV $>$ ACV & Douglas et al $\left.\right|^{18}$ \\
reduction in number of &
\end{tabular}

recurrences

Percentage with a decrease $\quad$ GEV/NV $=$ VACV Patel et $a^{20}$ in recurrence

Notes: In the comparison, we used the average clinical effects of all four GeneEden-VIR/Novirin dosages. Therefore, we also used the average clinical effects of all drug dosages in each of the five clinical studies. When the difference between our results and their results was $<10 \%$, we considered the results as equal.

Abbreviations: ACV, acyclovir; VACV, valacyclovir; FCV, famciclovir; GEV/NV, Gene-Eden-VIR/Novirin.
Our study did not attempt to measure the effect of Gene-Eden-VIR/Novirin on the herpes virus itself but the symptoms it causes. Yet, a better understanding of the GeneEden-VIR/Novirin mechanism of action is important. Such an understanding can be gleaned from some of the following in vitro studies that tested the effects of the Gene-Eden-VIR/ Novirin ingredients.

In one study, Laconi et al reported that glycyrrhizin, an active component of licorice, increased the production of Beclin 1 twofold more than rapamycin, a reference compound. ${ }^{26}$ Beclin 1 is an autophagy activator that was shown to inhibit the replication of HSV- $1 .{ }^{27}$ Laconi et al also showed that when glycyrrhizin was added to HeLa cells concurrently with HSV-1, glycyrrhizin had a strong anti-HSV-1 effect, whereas rapamycin did not. Other studies showed that licorice root decreased the adhesion of HSV-1 to host cells, and inhibited HSV-1 proliferation. ${ }^{28-30}$ Another study reported that epigallocatechin gallate, an active compound of green tea, interacted with tryptophan residues in HSV-1 surface proteins, and inhibited the attachment of the virus to its target cell by competing with heparin for HSV-1 binding. ${ }^{31}$ The same study also suggested that epigallocatechin gallate suppressed the HSV-1 replication by interrupting the late stages of the viral gene expression. Another study showed that quercetin activated two fuel-sensing molecules, AMPdependent kinase and Sirtuin, which are involved in survival pathways and neuroprotection. ${ }^{32}$ This activation increased the viability of HSV-1-infected neurons, and reduced the viral titer and expression of viral genes. The same study also showed that quercetin decreased the levels of caspase-3 cleaved and hyperphosphorylated tau, which are associated with HSV-1 infection. Another study showed that organic selenium decreased the activity of myeloperoxidase, and increased the TNF- $\alpha$ and IFN- $\gamma$ cytokines that play a beneficial role in the clearance of the HSV. ${ }^{33}$ The study also reported that selenium attenuated the increase of reactive species, including malondialdehyde, and the inhibition of superoxide dismutase caused by the virus. 
These in vitro studies show that the Gene-Eden-VIR/ Novirin ingredients have many effects on the HSV-1-infected cells. The additive effect of these mechanisms might explain how Gene-Eden-VIR/Novirin achieves its clinical objectives with the surprisingly low dosages of its five ingredients. Moreover, the many mechanisms highlight an important aspect of the Gene-Eden-VIR/Novirin therapy. While ACV, VACV, and FCV are single-target treatments, Gene-EdenVIR/Novirin is multi-target. In other words, while all three drugs inhibit the activity of a single molecule, the viral DNA polymerase, Gene-Eden-VIR/Novirin operates on many targets.

Our study has some methodological advantages. The cited clinical studies have a single duration of treatment. Specifically, Corey et a $\mathrm{l}^{10}$ assessed patients after 8 months of treatment. Douglas et al, ${ }^{18}$ Patel et al, ${ }^{20}$ and Mertz et al ${ }^{19}$ assessed patients after only 4 months of treatment, and Reitano et $\mathrm{al}^{12}$ assessed patients after 12 months of treatment. In contrast, our study tested the effect of the treatment over a wide range of durations, from 2 to 48 months, with an average of 12 months. Moreover, $28 \%$ of the participants in our study took Gene-Eden-VIR/Novirin for at least 2 years, and some, for as long as 4 years, a much longer duration of treatment that was tested in the cited clinical studies. The use of a range of durations, rather than a single duration, made it possible to test for a duration-of-treatment effect.

Another methodological advantage is using two types of questions to gather information about the frequency of outbreaks. The first type asked the participants to count the number of outbreaks, and the second asked them to rate the frequency of their outbreaks on a 1-7 scale. Using these two types, we verified the consistency in the participants' answers.

This post-marketing study did not include a placebo control. Placebo-controlled studies are regarded as the gold standard in medical research in premarketing clinical studies. However, except in rare cases, post-marketing studies do not use placebo controls. They use other controls recommended by the FDA. The FDA guidance lists six types of controls for both premarketing and post-marketing studies: 1) placebo concurrent control, 2) no-treatment concurrent control, 3) dose-response concurrent control, 4) active (positive) concurrent control, 5) external control (including historical control and baseline-controlled studies), and 6) multiple control groups. ${ }^{34}$ The Gene-Eden-VIR/Novirin post-marketing clinical study included three controls recommended by the FDA: a no-treatment concurrent control, a dose-response concurrent control, and a baseline control, a type of external control.
This study used PROs. These types of observations are used extensively in clinical trials for collecting clinical data. ${ }^{35}$ In fact, out of the 96,736 clinical trials registered in the ClinicalTrials.gov database between November 2007 and December 2013, 26,337, or 27\%, used at least one PRO measure. ${ }^{36}$ In addition, past studies showed that PROs had a significant role in the development and evaluation of new medicines. ${ }^{37}$ According to the FDA, PROs are a valid and valuable source for measuring the efficacy of new drugs. They are reliable enough to warrant an approval of a label claim for a new drug. From the years 1997 to 2002, the FDA approved 23 new drugs based on results obtained in studies that used only PRO end points. They include six antimigraine products $\left(\right.$ Amerge $^{\circledR}$, Ax-ert $\left.^{\circledR}\right)$, several antiepileptics $\left(\right.$ Gabitril ${ }^{\circledR}$, Keppra $^{\circledR}$ ), and a variety of other therapy classes (Tamiflu ${ }^{\circledR}$, Relenza $^{\circledR}$ ). The FDA regards PROs as a valid and valuable source of data. The scientific community also believes that PROs are valid and useful. Many major journals published clinical studies that use PROs. The trust of the FDA and the scientific community in PROs should convince the medical community, and specifically, doctors, to trust studies that use PROs when evaluating the benefits of new treatments.

A possible limitation of using PROs in our study is the subjective report of symptoms. To address this issue, we compared the symptoms reported by the participants to the standard signs and symptoms. The comparison clearly showed that the reported symptoms and those found in the literature overlap.

In summary, this study showed that suppressive treatment with Gene-Eden-VIR/Novirin safely decreased the frequency of genital herpes outbreaks. The study also showed that the clinical effects of Gene-Eden-VIR/Novirin are mostly better than those of ACV, VACV, and FCV.

\section{Disclosure}

The authors report no conflicts of interest in this work.

\section{References}

1. Johnston C, Corey L. Current concepts for genital herpes simplex virus infection: diagnostics and pathogenesis of genital tract shedding. Clin Microbiol Rev. 2016;29(1):149-161.

2. Gupta R, Warren T, Wald A. Genital herpes. Lancet. 2007;370(9605): 2127-2137.

3. Corey L, Adams HG, Brown ZA, Holmes KK. Genital herpes simplex virus infections: clinical manifestations, course, and complications. Ann Intern Med. 1983;98(6):958-972.

4. Lebrun-Vignes B, Bouzamondo A, Dupuy A, Guillaume JC, Lechat P, Chosidow O. A meta-analysis to assess the efficacy of oral antiviral treatment to prevent genital herpes outbreaks. J Am Acad Dermatol. 2007; 57(2):238-246.

5. Kukhanova MK, Korovina AN, Kochetkov SN. Human herpes simplex virus: life cycle and development of inhibitors. Biochemistry (Mosc) 2014;79(13):1635-1652. 
6. Mattison HR, Reichman RC, Benedetti J, et al. Double-blind, placebocontrolled trial comparing long-term suppressive with short-term oral acyclovir therapy for management of recurrent genital herpes. Am J Med. 1988;85(2A):20-25.

7. Reichman RC, Badger GJ, Mertz GJ, et al. Treatment of recurrent genital herpes simplex infections with oral acyclovir. JAMA. 1984;251(16): 2103-2107.

8. Tyring S, Wald A, Zadeikis N, Dhadda S, TakenouchiK, Rorig R. ASP2151 for the treatment of genital herpes: a randomized, double-blind, placeboand valacyclovir-controlled, dose-finding study. J Infect Dis. 2012; 205(7):1100-1110.

9. Aoki FY, Tyring S, Diaz-Mitoma F, Gross G, Gao J, Hamed K. Singleday, patient-initiated famciclovir therapy for recurrent genital herpes: a randomized, double-blind, placebo-controlled trial. Clin Infect Dis. 2006;42(1):8-13.

10. Corey L, Wald A, Patel R, et al. Once-daily valacyclovir to reduce the risk of transmission of genital herpes. N Engl J Med. 2004;350(1):11-20.

11. Cernik C, Gallina K, Brodell RT. The treatment of herpes simplex infections: an evidence-based review. Arch Intern Med. 2008;168(11): 1137.

12. Reitano M, Tyring S, Lang W, et al. Valaciclovir for the suppression of recurrent genital herpes simplex virus infection: a large-scale dose range-finding study. International Valaciclovir HSV Study Group. J Infect Dis. 1998;178(3):603-610.

13. Polansky H, Javaherian A. 3-Econsystems: microRNAs, receptors, and latent viruses: some insights biology can gain from economic theory. Front Microbiol. 2016;7:369.

14. Polansky H, Javaherian A. Commentary: the unliganded glucocorticoid receptor positively regulates the tumor suppressor gene BRCA1 through GABP beta. Front Cell Infect Microbiol. 2015;5:66.

15. Polansky H. Microcompetition with Foreign DNA and the Origin of Chronic Disease. New York: CBCD Publishing; 2003.

16. Polansky H, Itzkovitz E. Gene-Eden-VIR is antiviral: results of a post marketing clinical study. Pharmacol Pharm. 2013;4(6A):1-8.

17. Polansky H, Itzkovitz E. Gene-Eden-VIR decreased physical and mental fatigue in a post marketing clinical study that followed FDA guidelines; results support microcompetition theory. Pharmacol Pharm. 2014;5(3):280-290.

18. Douglas JM, Critchlow C, Benedetti J, et al. A double-blind study of oral acyclovir for suppression of recurrences of genital herpes simplex virus infection. $N$ Engl J Med. 1984;310(24):1551-1556.

19. Mertz GJ, Loveless MO, Levin MJ, et al. Oral famciclovir for suppression of recurrent genital herpes simplex virus infection in women. A multicenter, double-blind, placebo-controlled trial. Collaborative Famciclovir Genital Herpes Research Group. Arch Intern Med. 1997; 157(3):343-349.

20. Patel R, Bodsworth NJ, Woolley P, et al. Valaciclovir for the suppression of recurrent genital HSV infection: a placebo controlled study of once daily therapy. International Valaciclovir HSV Study Group. Genitourin Med. 1997;73(2):105-109.

21. Yavuz BB, Cankurtaran M, Halil M, Dagli N, Kirkpantur A. Renal dysfunction after oral acyclovir treatment in a geriatric woman: a case report. Scand J Infect Dis. 2005;37(8):611-613.

22. Becker BN, Fall P, Hall C, et al. Rapidly progressive acute renal failure due to acyclovir: case report and review of the literature. Am J Kidney Dis. 1993;22(4):611-615.
23. Berry L, Venkatesan P. Aciclovir-induced neurotoxicity: utility of CSF and serum CMMG levels in diagnosis. J Clin Virol. 2014;61(4): 608-610.

24. Le Cleach L, Trinquart L, Do G, et al. Oral antiviral therapy for prevention of genital herpes outbreaks in immunocompetent and nonpregnant patients. Cochrane Database Syst Rev. 2014;8:CD009036.

25. Lam NN, Weir MA, Yao Z, et al. Risk of acute kidney injury from oral acyclovir: a population-based study. Am J Kidney Dis. 2013;61(5): 723-729.

26. Laconi S, Madeddu MA, Pompei R. Autophagy activation and antiviral activity by a licorice triterpene. Phytother Res. 2014;28(12): 1890-1892.

27. Wang L, Yang R, Yuan B, Liu Y, Liu C. The antiviral and antimicrobial activities of licorice, a widely-used Chinese herb. Acta Pharm Sin B. 2015;5(4):310-315.

28. Sabouri GM, Mohammadi A, Safiallahy S, Faradmal J, Azizi M, Ahmadvand Z. The effect of aqueous extract of glycyrrhiza glaba on herpes simplex virus 1. Jundishapur J Microbiol. 2014;7(7):e11616.

29. Hung PY, Ho BC, Lee SY, et al. Houttuynia cordata targets the beginning stage of herpes simplex virus infection. PLoS One. 2015; 10(2):e0115475.

30. Orihara Y, Hamamoto H, Kasuga H, Shimada T, Kawaguchi Y, Sekimizu K. A silkworm baculovirus model for assessing the therapeutic effects of antiviral compounds: characterization and application to the isolation of antivirals from traditional medicines. J Gen Virol. 2008;89(Pt 1):188-194.

31. Colpitts CC, Schang LM. A small molecule inhibits virion attachment to heparin sulfate- or sialic acid-containing glycans. J Virol. 2014; 88(14):7806-7817.

32. Leyton L, Hott M, Acuna F, et al. Nutraceutical activators of AMPK/ Sirt1 axis inhibit viral production and protect neurons from neurodegenerative events triggered during HSV-1 infection. Virus Res. 2015; 205:63-72.

33. Sartori G, Jardim NS, Marcondes Sari MH, et al. Antiviral action of diphenyl diselenide on herpes simplex virus 2 infection in female BALB/c mice. J Cell Biochem. 2016;117(7):1638-1648.

34. US Department of Health and Human Services, Food and Drug Administration, Center for Drug Evaluation and Research (CDER). Guidance for industry, E 10 choice of control group and related issues in clinical trials. 2001. Available from: http://www.fda.gov/downloads/drugs/ guidancecomplianceregulatoryinformation/guidances/ucm073139.pdf. Accessed August 16, 2016.

35. Calvert M, Kyte D, Duffy H, et al. Patient-reported outcome (PRO) assessment in clinical trials: a systematic review of guidance for trial protocol writers. PLoS One. 2014;9(10):e110216.

36. Vodicka E, Kim K, Devine EB, Gnanasakthy A, Scoggins JF, Patrick DL. Inclusion of patient-reported outcome measures in registered clinical trials: evidence from ClinicalTrials.gov (2007-1013). Contemp Clin Trials. 2015;43:1-9.

37. Willke RJ, Burke LB, Erickson P. Measuring treatment impact: a review of patient-reported outcomes and other efficacy endpoints in approved product labels. Control Clin Trials. 2004;25(6):535-552.
Drug Design, Development and Therapy

\section{Publish your work in this journal}

Drug Design, Development and Therapy is an international, peerreviewed open-access journal that spans the spectrum of drug design and development through to clinical applications. Clinical outcomes, patient safety, and programs for the development and effective, safe, and sustained use of medicines are the features of the journal, which

\section{Dovepress}

has also been accepted for indexing on PubMed Central. The manuscript management system is completely online and includes a very quick and fair peer-review system, which is all easy to use. Visit http://www.dovepress.com/testimonials.php to read real quotes from published authors. 